\title{
The virtual and universal braids
}

\author{
by \\ Valerij G. Bardakov (Novosibirsk)
}

\begin{abstract}
We study the structure of the virtual braid group. It is shown that the virtual braid group is a semi-direct product of the virtual pure braid group and the symmetric group. Also, it is shown that the virtual pure braid group is a semi-direct product of free groups. From these results we obtain a normal form of words in the virtual braid group. We introduce the concept of a universal braid group. This group contains the classical braid group and has as quotients the singular braid group, virtual braid group, welded braid group, and classical braid group.
\end{abstract}

Recently some generalizations of classical knots and links were defined and studied: singular links [20,5], virtual links [15, 12] and welded links [10].

One of the ways to study classical links is to study the braid group. Singular braids [1, 5], virtual braids [15, 21], welded braids [10] were defined similarly to the classical braid group. A theorem of A. A. Markov [4, Ch. 2.2 ] reduces the problem of classification of links to some algebraic problems of the theory of braid groups. These problems include the word problem and the conjugacy problem. There are generalizations of Markov's theorem to singular links [11], virtual links, and welded links [14].

There are some different ways to solve the word problem for the singular braid monoid and singular braid group [8, 7, 22]. The solution of the word problem for the welded braid group follows from the fact that this group is a subgroup of the automorphism group of the free group [10]. A normal form of words in the welded braid group was constructed in [13].

In this paper we study the structure of the virtual braid group $V B_{n}$. This is similar to the classical braid group $B_{n}$ and welded braid group $W B_{n}$. The group $V B_{n}$ contains the normal subgroup $V P_{n}$ which is called the virtual

2000 Mathematics Subject Classification: Primary 20F36; Secondary 20F05, 20F10.

Key words and phrases: knot theory, singular knot, virtual knot, braid group, singular braid monoid, free groups, automorphism, word problem.

The author was supported in part by the Russian Foundation for Basic Research (grant 02-01-01118). 
pure braid group. The quotient group $V B_{n} / V P_{n}$ is isomorphic to the symmetric group $S_{n}$. We find generators and defining relations of $V P_{n}$. Since $V B_{n}$ is a semi-direct product of $V P_{n}$ and $S_{n}$, we should study the structure of $V P_{n}$. It will be proved that $V P_{n}$ is representable as the following semi-direct product:

$$
V P_{n}=V_{n-1}^{*} \rtimes V P_{n-1}=V_{n-1}^{*} \rtimes\left(V_{n-2}^{*} \rtimes\left(\cdots \rtimes\left(V_{2}^{*} \rtimes V_{1}^{*}\right)\right) \cdots\right),
$$

where $V_{i}^{*}$ is some (in general infinitely generated for $i>1$ ) free subgroup of $V P_{n}$. From this result it follows that there exists a normal form of words in $V B_{n}$.

In the last section we define the universal braid group $U B_{n}$ which contains the braid group $B_{n}$ and has as quotients the singular braid group $S G_{n}$, the virtual braid group $V B_{n}$, the welded braid group $W B_{n}$, and the braid group $B_{n}$. It is known [10] that $V B_{n}$ has as its quotient the group $W B_{n}$. It will be proved that the quotient homomorphism maps $V P_{n}$ into the welded pure braid group $W P_{n}$. This homomorphism agrees with the decomposition of this group into the semi-direct product given by Theorem 2 and by $[2,3]$.

By Artin's theorem, the group $B_{n}$ is embedded into the automorphism group $\operatorname{Aut}\left(F_{n}\right)$ of the free group $F_{n}$. In [10] it was proved that $W B_{n}$ is also embedded into $\operatorname{Aut}\left(F_{n}\right)$. It is not known if $S G_{n}$ and $V B_{n}$ are embedded into $\operatorname{Aut}\left(F_{n}\right)$.

Acknowledgments. I am grateful to Joanna Kania-Bartoszyńska, Józef Przytycki, Paweł Traczyk, and Bronisław Wajnryb for the invitation to participate in the very interesting and well organized Mini-semester on Knot Theory (Poland, July, 2003). I would also like to thank Vladimir Vershinin and Andrei Vesnin for their interest in this work. Special thanks go to the participants of the seminar "Evariste Galois" at the Novosibirsk State University for their kind interest in my work.

1. Different classes of braids and their properties. In this section we recall some known facts about braid groups, singular braid monoids, virtual braid groups and welded braid groups (see references from the introduction).

1.1. The braid group and the group of conjugating automorphisms. The braid group $B_{n}, n \geq 2$, on $n$ strings can be defined as the group generated by $\sigma_{1}, \ldots, \sigma_{n-1}$ (see Fig. 1 ) with the defining relations

$$
\begin{aligned}
\sigma_{i} \sigma_{i+1} \sigma_{i} & =\sigma_{i+1} \sigma_{i} \sigma_{i+1}, & & i=1, \ldots, n-2, \\
\sigma_{i} \sigma_{j} & =\sigma_{j} \sigma_{i}, & & |i-j| \geq 2 .
\end{aligned}
$$

There exists a homomorphism of $B_{n}$ onto the symmetric group $S_{n}$ on $n$ letters. This homomorphism maps $\sigma_{i}$ to the transposition $(i, i+1), i=$ $1, \ldots, n-1$. Its kernel is called the pure braid group and denoted by $P_{n}$. The 

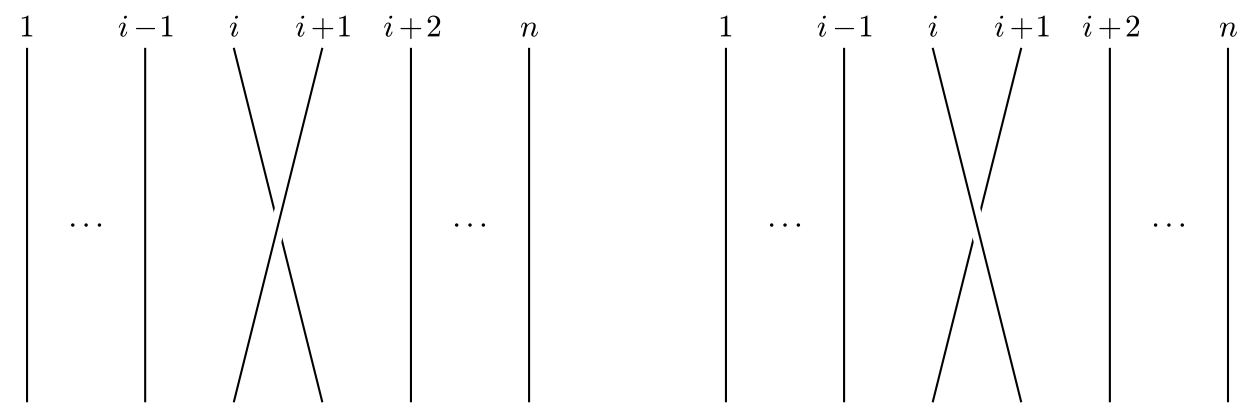

Fig. 1. Geometric braids representing $\sigma_{i}$ and $\sigma_{i}^{-1}$

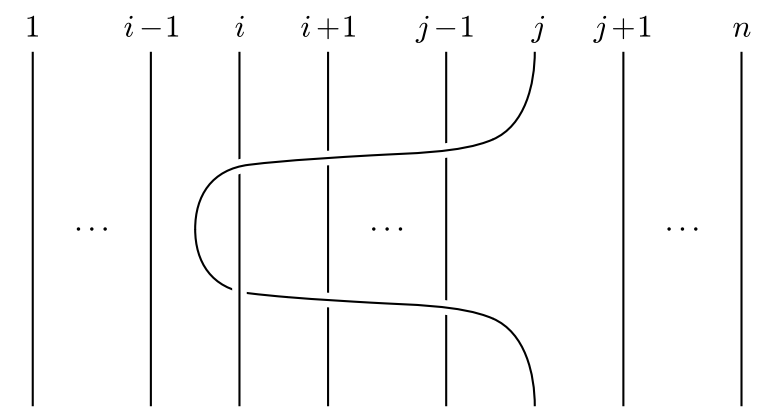

Fig. 2. The geometric braid $a_{i j}$

group $P_{n}$ is generated by $a_{i j}, 1 \leq i<j \leq n$ (see Fig. 2). These generators can be expressed by the generators of $B_{n}$ as follows:

$$
\begin{gathered}
a_{i, i+1}=\sigma_{i}^{2} \\
a_{i j}=\sigma_{j-1} \sigma_{j-2} \ldots \sigma_{i+1} \sigma_{i}^{2} \sigma_{i+1}^{-1} \ldots \sigma_{j-2}^{-1} \sigma_{j-1}^{-1}, \quad i+1<j \leq n .
\end{gathered}
$$

The group $P_{n}$ is the semi-direct product of the normal subgroup $U_{n}$ which is a free group with free generators $a_{1 n}, a_{2 n}, \ldots, a_{n-1, n}$, and $P_{n-1}$. Similarly, $P_{n-1}$ is the semi-direct product of the free group $U_{n-1}$ with free generators $a_{1, n-1}, a_{2, n-1}, \ldots, a_{n-2, n-1}$ and $P_{n-2}$, and so on. Therefore, $P_{n}$ is decomposable (see [17]) into the following semi-direct product:

$$
P_{n}=U_{n} \rtimes\left(U_{n-1} \rtimes\left(\cdots \rtimes\left(U_{3} \rtimes U_{2}\right)\right) \cdots\right), \quad U_{i} \simeq F_{i-1}, i=2, \ldots, n .
$$

The group $B_{n}$ in the faithful representation in the group $\operatorname{Aut}\left(F_{n}\right)$ of automorphisms of the free group $F_{n}=\left\langle x_{1}, \ldots, x_{n}\right\rangle$. In this case the generator $\sigma_{i}, i=1, \ldots, n-1$, defines the automorphism

$$
\sigma_{i}:\left\{\begin{array}{l}
x_{i} \mapsto x_{i} x_{i+1} x_{i}^{-1}, \\
x_{i+1} \mapsto x_{i}, \\
x_{l} \mapsto x_{l}, \quad l \neq i, i+1 .
\end{array}\right.
$$


By a theorem of Artin [4, Theorem 1.9], an automorphism $\beta \in \operatorname{Aut}\left(F_{n}\right)$ lies in $B_{n}$ if and only if $\beta$ satisfies the following conditions:
(i) $\beta\left(x_{i}\right)=a_{i}^{-1} x_{\pi(i)} a_{i}, \quad 1 \leq i \leq n$,
(ii) $\beta\left(x_{1} \cdots x_{n}\right)=x_{1} \cdots x_{n}$,

where $\pi$ is a permutation from $S_{n}$ and $a_{i} \in F_{n}$.

An automorphism is called a conjugating automorphism (or a permutation-conjugating automorphism according to the terminology of [10]) if it satisfies condition (i). The group $C_{n}$ of conjugating automorphisms is generated by $\sigma_{i}$ and the automorphisms $\alpha_{i}, i=1, \ldots, n-1$, where

$$
\alpha_{i}:\left\{\begin{array}{l}
x_{i} \mapsto x_{i+1}, \\
x_{i+1} \mapsto x_{i}, \\
x_{l} \mapsto x_{l}, \quad l \neq i, i+1 .
\end{array}\right.
$$

It is not hard to see that the automorphisms $\alpha_{i}$ generate the symmetric group $S_{n}$ and, hence, satisfy the following relations:

$$
\begin{aligned}
\alpha_{i} \alpha_{i+1} \alpha_{i} & =\alpha_{i+1} \alpha_{i} \alpha_{i+1}, & & i=1, \ldots, n-2, \\
\alpha_{i} \alpha_{j} & =\alpha_{j} \alpha_{i}, & & |i-j| \geq 2, \\
\alpha_{i}^{2} & =1, & & i=1, \ldots, n-1 .
\end{aligned}
$$

The group $C_{n}$ is defined by relations (1)-(2) of $B_{n}$, relations (3)-(5) of $S_{n}$, and the mixed relations (see $[10,19]$ )

$$
\begin{aligned}
\alpha_{i} \sigma_{j} & =\sigma_{j} \alpha_{i}, & & |i-j| \geq 2, \\
\sigma_{i} \alpha_{i+1} \alpha_{i} & =\alpha_{i+1} \alpha_{i} \sigma_{i+1}, & & i=1, \ldots, n-2, \\
\sigma_{i+1} \sigma_{i} \alpha_{i+1} & =\alpha_{i} \sigma_{i+1} \sigma_{i}, & & i=1, \ldots, n-2 .
\end{aligned}
$$

If we consider the group generated by the automorphisms $\varepsilon_{i j}, 1 \leq i \neq$ $j \leq n$, where

$$
\varepsilon_{i j}: \begin{cases}x_{i} \mapsto x_{j}^{-1} x_{i} x_{j}, & i \neq j, \\ x_{l} \mapsto x_{l}, & l \neq i,\end{cases}
$$

then we get the group $C b_{n}$ of basis-conjugating automorphisms. The elements of satisfy condition (i) for the identical permutation $\pi$, i.e., map each generator $x_{i}$ to the conjugating element. J. McCool [18] proved that $C b_{n}$ is defined by the relations (from now on, different letters stand for different indices)

$$
\begin{aligned}
\varepsilon_{i j} \varepsilon_{k l} & =\varepsilon_{k l} \varepsilon_{i j}, \\
\varepsilon_{i j} \varepsilon_{k j} & =\varepsilon_{k j} \varepsilon_{i j}, \\
\left(\varepsilon_{i j} \varepsilon_{k j}\right) \varepsilon_{i k} & =\varepsilon_{i k}\left(\varepsilon_{i j} \varepsilon_{k j}\right) .
\end{aligned}
$$

The group $C_{n}$ is representable as the semi-direct product $C_{n}=C b_{n} \rtimes S_{n}$, where $S_{n}$ is generated by the automorphisms $\alpha_{1}, \ldots, \alpha_{n-1}$. The following 
equalities hold (see [19]):

$$
\begin{array}{cc}
\varepsilon_{i, i+1}=\alpha_{i} \sigma_{i}^{-1}, \quad \varepsilon_{i+1, i}=\sigma_{i}^{-1} \alpha_{i}, & \\
\varepsilon_{i j}=\alpha_{j-1} \alpha_{j-2} \cdots \alpha_{i+1} \varepsilon_{i, i+1} \alpha_{i+1} \cdots \alpha_{j-2} \alpha_{j-1}, & i<j, \\
\varepsilon_{j i}=\alpha_{j-1} \alpha_{j-2} \cdots \alpha_{i+1} \alpha_{i} \varepsilon_{i, i+1} \alpha_{i} \alpha_{i+1} \cdots \alpha_{j-2} \alpha_{j-1}, & i<j .
\end{array}
$$

The structure of $C b_{n}$ was studied in $[2,3]$. It was proved that $C b_{n}, n \geq 2$, is decomposable into the semi-direct product

$$
C b_{n}=D_{n-1} \rtimes\left(D_{n-2} \rtimes\left(\cdots \rtimes\left(D_{2} \rtimes D_{1}\right)\right) \cdots\right)
$$

of subgroups $D_{i}, i=1, \ldots, n-1$, generated by $\varepsilon_{i+1,1}, \varepsilon_{i+1,2}, \ldots, \varepsilon_{i+1, i}, \varepsilon_{1, i+1}$, $\varepsilon_{2, i+1}, \ldots, \varepsilon_{i, i+1}$. The elements $\varepsilon_{i+1,1}, \varepsilon_{i+1,2}, \ldots, \varepsilon_{i+1, i}$ generate a free group of rank $i$. The elements $\varepsilon_{1, i+1}, \varepsilon_{2, i+1}, \ldots, \varepsilon_{i, i+1}$ generate a free abelian group of rank $i$.

The pure braid group $P_{n}$ is contained in $C b_{n}$ and the generators of $P_{n}$ can be written in the form

$$
\begin{gathered}
a_{i, i+1}=\varepsilon_{i, i+1}^{-1} \varepsilon_{i+1, i}^{-1}, \quad i=1, \ldots, n-1, \\
a_{i j}=\varepsilon_{j-1, i} \varepsilon_{j-2, i} \ldots \varepsilon_{i+1, i}\left(\varepsilon_{i j}^{-1} \varepsilon_{j i}^{-1}\right) \varepsilon_{i+1, i}^{-1} \ldots \varepsilon_{j-2, i}^{-1} \varepsilon_{j-1, i}^{-1} \\
=\varepsilon_{j-1, j}^{-1} \varepsilon_{j-2, j}^{-1} \ldots \varepsilon_{i+1, j}^{-1}\left(\varepsilon_{i j}^{-1} \varepsilon_{j i}^{-1}\right) \varepsilon_{i+1, j} \ldots \varepsilon_{j-2, j} \varepsilon_{j-1, j}, \quad 2 \leq i+1<j \leq n .
\end{gathered}
$$

1.2. The singular braid monoid. The Baez-Birman monoid $[1,5]$ or the singular braid monoid $S B_{n}$ is generated (as a monoid) by elements $\sigma_{i}, \sigma_{i}^{-1}$, $\tau_{i}, i=1, \ldots, n-1$. The elements $\sigma_{i}, \sigma_{i}^{-1}$ generate the braid group $B_{n}$. The generators $\tau_{i}$ satisfy the defining relations

$$
\tau_{i} \tau_{j}=\tau_{j} \tau_{i}, \quad|i-j| \geq 2,
$$

and the other relations are mixed:

$$
\begin{aligned}
\tau_{i} \sigma_{j} & =\sigma_{j} \tau_{i}, & & |i-j| \geq 2, \\
\tau_{i} \sigma_{i} & =\sigma_{i} \tau_{i}, & & i=1, \ldots, n-1, \\
\sigma_{i} \sigma_{i+1} \tau_{i} & =\tau_{i+1} \sigma_{i} \sigma_{i+1}, & & i=1, \ldots, n-2, \\
\sigma_{i+1} \sigma_{i} \tau_{i+1} & =\tau_{i} \sigma_{i+1} \sigma_{i}, & & i=1, \ldots, n-2 .
\end{aligned}
$$

In [9] it was proved that the singular braid monoid $S B_{n}$ is embedded into the group $S G_{n}$ which is called the singular braid group and has the same defining relations as $S B_{n}$.

1.3. The virtual braid group and welded braid group. The virtual braid group $V B_{n}$ was introduced in [15]. In [21] a shorter system of defining relations was found (see below). The group $V B_{n}$ is generated by $\sigma_{i}, \varrho_{i}$, $i=1, \ldots, n-1$ (see Fig. 3 ).

The elements $\sigma_{i}$ generate the braid group $B_{n}$ with defining relations (1)(2) and the elements $\varrho_{i}$ generate the symmetric group $S_{n}$ which is defined 


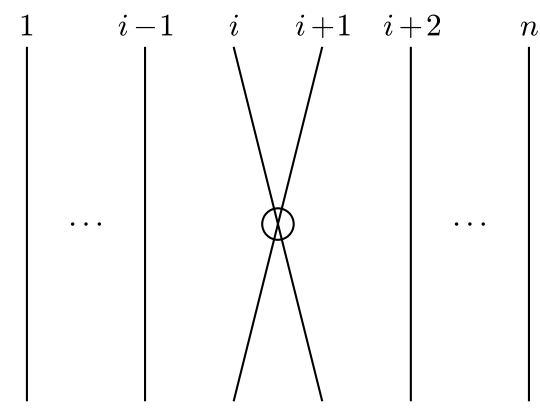

Fig. 3. The geometric virtual braid $\varrho_{i}$

by the relations

$$
\begin{aligned}
\varrho_{i} \varrho_{i+1} \varrho_{i} & =\varrho_{i+1} \varrho_{i} \varrho_{i+1}, & & i=1, \ldots, n-2, \\
\varrho_{i} \varrho_{j} & =\varrho_{j} \varrho_{i}, & & |i-j| \geq 2, \\
\varrho_{i}^{2} & =1, & & i=1, \ldots, n-1 .
\end{aligned}
$$

The other relations are mixed:

$$
\begin{aligned}
\sigma_{i} \varrho_{j} & =\varrho_{j} \sigma_{i}, & & |i-j| \geq 2, \\
\varrho_{i} \varrho_{i+1} \sigma_{i} & =\sigma_{i+1} \varrho_{i} \varrho_{i+1}, & & i=1, \ldots, n-2 .
\end{aligned}
$$

Note that the last relation is equivalent to

$$
\varrho_{i+1} \varrho_{i} \sigma_{i+1}=\sigma_{i} \varrho_{i+1} \varrho_{i} .
$$

In [12] it was proved that the relations

$$
\varrho_{i} \sigma_{i+1} \sigma_{i}=\sigma_{i+1} \sigma_{i} \varrho_{i+1}, \quad \varrho_{i+1} \sigma_{i} \sigma_{i+1}=\varrho_{i} \sigma_{i+1} \sigma_{i}
$$

are not satisfied in $V B_{n}$.

The welded braid group $W B_{n}$ was introduced in [10]. This group is generated by $\sigma_{i}, \alpha_{i}, i=1, \ldots, n-1$. The elements $\sigma_{i}$ generate the braid group $B_{n}$. The elements $\alpha_{i}$ generate the symmetric group $S_{n}$ and the following mixed relations hold:

$$
\begin{aligned}
\alpha_{i} \sigma_{j} & =\sigma_{j} \alpha_{i}, & & |i-j| \geq 2, \\
\sigma_{i} \alpha_{i+1} \alpha_{i} & =\alpha_{i+1} \alpha_{i} \sigma_{i+1}, & & i=1, \ldots, n-2, \\
\sigma_{i+1} \sigma_{i} \alpha_{i+1} & =\alpha_{i} \sigma_{i+1} \sigma_{i}, & & i=1, \ldots, n-2 .
\end{aligned}
$$

In [10] it was proved that $W B_{n}$ is isomorphic to the group $C_{n}$ of conjugating automorphisms.

Comparing the defining relations of $V B_{n}$ with the defining relations of $W B_{n}$, we see that $W B_{n}$ can be obtained from $V B_{n}$ by adding a new relation. Therefore, there exists a homomorphism

$$
\varphi_{V W}: V B_{n} \rightarrow W B_{n}
$$


taking $\sigma_{i}$ to $\sigma_{i}$ and $\varrho_{i}$ to $\alpha_{i}$ for all $i$. Hence, $W B_{n}$ is the homomorphic image of $V B_{n}$.

In [10] it was proved that the relation (symmetric to (23))

$$
\sigma_{i+1} \alpha_{i} \alpha_{i+1}=\alpha_{i} \alpha_{i+1} \sigma_{i},
$$

is true in $W B_{n}$. But the following relation does not hold:

$$
\alpha_{i+1} \sigma_{i} \sigma_{i+1}=\sigma_{i} \sigma_{i+1} \alpha_{i} .
$$

Linear representations of $V B_{n}$ and $W B_{n}$ by matrices from $\mathrm{GL}_{n}\left(\mathbb{Z}\left[t, t^{-1}\right]\right)$ which extend the well known Burau representation were constructed in [21]. A linear representation of $C_{n} \simeq W B_{n}$ was constructed in [3]. This representation extends (with some conditions on parameters) the known LawrenceKrammer representation.

\section{Generators and defining relations of the virtual pure braid} group. In this section we introduce a virtual pure braid group and find its generators and defining relations.

Define the map $\nu: V B_{n} \rightarrow S_{n}$ by its action on the generators:

$$
\nu\left(\sigma_{i}\right)=\nu\left(\varrho_{i}\right)=\varrho_{i}, \quad i=1, \ldots, n-1,
$$

where $S_{n}$ is the group generated by $\varrho_{i}$. Then $\operatorname{ker}(\nu)$ is called the virtual pure braid group and denoted by $V P_{n}$. It is clear that $V P_{n}$ is a normal subgroup of index $n$ ! of $V B_{n}$. Moreover, since $V P_{n} \cap S_{n}=e$ and $V B_{n}=V P_{n} \cdot S_{n}$, we have $V B_{n}=V P_{n} \rtimes S_{n}$.

Define

$$
\begin{gathered}
\lambda_{i, i+1}=\varrho_{i} \sigma_{i}^{-1}, \quad \lambda_{i+1, i}=\varrho_{i} \lambda_{i, i+1} \varrho_{i}=\sigma_{i}^{-1} \varrho_{i}, \quad i=1, \ldots, n-1, \\
\lambda_{i j}=\varrho_{j-1} \varrho_{j-2} \cdots \varrho_{i+1} \lambda_{i, i+1} \varrho_{i+1} \cdots \varrho_{j-2} \varrho_{j-1}, \\
\lambda_{j i}=\varrho_{j-1} \varrho_{j-2} \cdots \varrho_{i+1} \lambda_{i+1, i} \varrho_{i+1} \cdots \varrho_{j-2} \varrho_{j-1}, \quad 1 \leq i<j-1 \leq n-1 .
\end{gathered}
$$

Obviously, all these elements belong to $V P_{n}$. Their geometric interpretation is shown in Figs. 4 and 5.

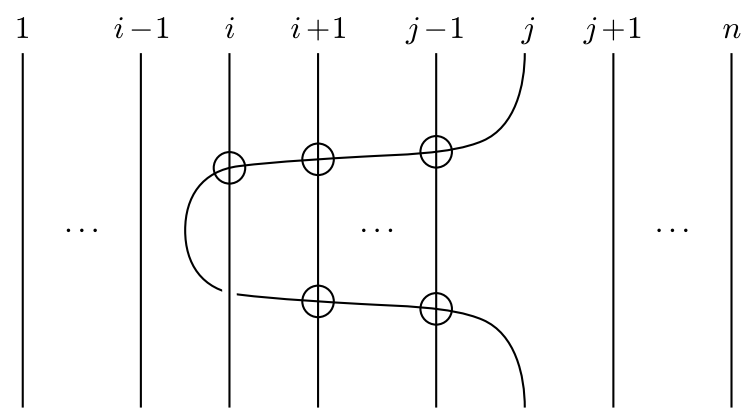

Fig. 4. The geometric virtual braid $\lambda_{i j}(1 \leq i<j \leq n)$ 


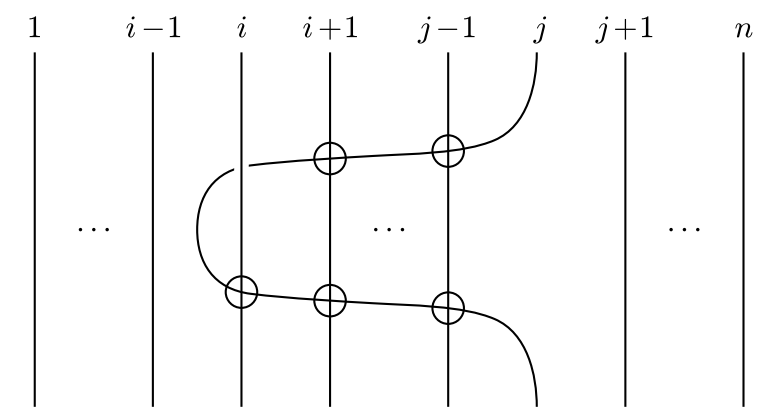

Fig. 5. The geometric virtual braid $\lambda_{j i}(1 \leq i<j \leq n)$

Lemma 1. Let $1 \leq i<j \leq n$. The following conjugation rules are satisfied in $V B_{n}$ :

(i) for $k<i-1, i<k<j-1$ and $k>j$,

$$
\varrho_{k} \lambda_{i j} \varrho_{k}=\lambda_{i j}, \varrho_{k} \lambda_{j i} \varrho_{k}=\lambda_{j i}
$$

(ii) $\varrho_{i-1} \lambda_{i j} \varrho_{i-1}=\lambda_{i-1, j}, \varrho_{i-1} \lambda_{j i} \varrho_{i-1}=\lambda_{j, i-1}$;

(iii) for $i<j-1$,

$$
\begin{array}{ll}
\varrho_{i} \lambda_{i, i+1} \varrho_{i}=\lambda_{i+1, i}, & \varrho_{i} \lambda_{i j} \varrho_{i}=\lambda_{i+1, j}, \\
\varrho_{i} \lambda_{i+1, i} \varrho_{i}=\lambda_{i, i+1}, & \varrho_{i} \lambda_{j i} \varrho_{i}=\lambda_{j, i+1} ;
\end{array}
$$

(iv) for $i+1<j$,

$$
\varrho_{j-1} \lambda_{i j} \varrho_{j-1}=\lambda_{i, j-1}, \quad \varrho_{j-1} \lambda_{j i} \varrho_{j-1}=\lambda_{j-1, i}
$$

(v) $\varrho_{j} \lambda_{i j} \varrho_{j}=\lambda_{i, j+1}, \quad \varrho_{j} \lambda_{j i} \varrho_{j}=\lambda_{j+1, i}$.

Proof. We consider only the rules involving $\lambda_{i j}$ for $i<j$ (the remaining ones can be considered analogously). Recall that

$$
\lambda_{i j}=\varrho_{j-1} \varrho_{j-2} \cdots \varrho_{i+1} \lambda_{i, i+1} \varrho_{i+1} \cdots \varrho_{j-2} \varrho_{j-1} .
$$

If $k<i-1$ or $k>j$ then $\varrho_{k}$ is permutable with $\varrho_{i}, \varrho_{i+1}, \ldots, \varrho_{j-1}$ in view of relation (18) and with $\sigma_{i}$ in view of relation (20). Hence, $\varrho_{k}$ is permutable with $\lambda_{i j}$.

Let $i<k<j-1$. Then $\varrho_{k} \lambda_{i j} \varrho_{k}=\varrho_{k}\left(\varrho_{j-1} \cdots \varrho_{k+2} \varrho_{k+1} \varrho_{k} \cdots \varrho_{i+1} \lambda_{i, i+1} \varrho_{i+1} \cdots \varrho_{k} \varrho_{k+1} \varrho_{k+2} \cdots \varrho_{j-1}\right) \varrho_{k}$.

Permuting $\varrho_{k}$ and $\lambda_{i, i+1}$ whenever possible, we get

$$
\varrho_{j-1} \cdots \varrho_{k+2}\left(\varrho_{k} \varrho_{k+1} \varrho_{k}\right) \cdots \varrho_{i+1} \lambda_{i, i+1} \varrho_{i+1} \cdots\left(\varrho_{k} \varrho_{k+1} \varrho_{k}\right) \varrho_{k+2} \cdots \varrho_{j-1} \text {. }
$$

Using the relation $\varrho_{k} \varrho_{k+1} \varrho_{k}=\varrho_{k+1} \varrho_{k} \varrho_{k+1}$, we rewrite the last formula as follows:

$$
\begin{aligned}
\varrho_{j-1} \cdots \varrho_{k+1} \varrho_{k}\left(\varrho_{k+1} \varrho_{k-1} \cdots \varrho_{i+1} \lambda_{i, i+1} \varrho_{i+1} \cdots \varrho_{k-1} \varrho_{k+1}\right) \\
\times \varrho_{k} \varrho_{k+1} \cdots \varrho_{j-1}=\varrho_{j-1} \cdots \varrho_{k}\left(\varrho_{k+1} \lambda_{i, k} \varrho_{k+1}\right) \varrho_{k} \cdots \varrho_{j-1} .
\end{aligned}
$$


In view of the case considered earlier, we have

$$
\varrho_{k+1} \lambda_{i k} \varrho_{k+1}=\lambda_{i k}
$$

and, hence,

$$
\varrho_{j-1} \cdots \varrho_{k}\left(\varrho_{k+1} \lambda_{i k} \varrho_{k+1}\right) \varrho_{k} \cdots \varrho_{j-1}=\lambda_{i j} .
$$

Thus, the first rule of (i) is proven.

(ii) Consider

$$
\varrho_{i-1} \lambda_{i j} \varrho_{i-1}=\varrho_{i-1}\left(\varrho_{j-1} \varrho_{j-2} \cdots \varrho_{i+1} \lambda_{i, i+1} \varrho_{i+1} \cdots \varrho_{j-2} \varrho_{j-1}\right) \varrho_{i-1} .
$$

Using (18), let us permute $\varrho_{i-1}$ and $\lambda_{i, i+1}$ whenever possible. We get

$$
\varrho_{i-1} \lambda_{i j} \varrho_{i-1}=\varrho_{j-1} \ldots \varrho_{i+2} \varrho_{i+1}\left(\varrho_{i-1} \lambda_{i, i+1} \varrho_{i-1}\right) \varrho_{i+1} \varrho_{i+2} \ldots \varrho_{j-2} .
$$

The expression in brackets can be rewritten as

$$
\varrho_{i-1} \lambda_{i, i+1} \varrho_{i-1}=\varrho_{i-1} \varrho_{i} \sigma_{i}^{-1} \varrho_{i-1}=\varrho_{i-1} \varrho_{i} \sigma_{i}^{-1} \varrho_{i-1} \varrho_{i} \varrho_{i} .
$$

Using the relation $\sigma_{i}^{-1} \varrho_{i-1} \varrho_{i}=\varrho_{i-1} \varrho_{i} \sigma_{i-1}^{-1}$ (following from (21)) and (18), (19), we obtain

$$
\begin{aligned}
\varrho_{i-1} \varrho_{i}\left(\sigma_{i}^{-1} \varrho_{i-1} \varrho_{i}\right) \varrho_{i} & =\varrho_{i-1}\left(\varrho_{i} \varrho_{i-1} \varrho_{i}\right) \sigma_{i-1}^{-1} \varrho_{i} \\
& =\left(\varrho_{i-1} \varrho_{i-1}\right) \varrho_{i} \varrho_{i-1} \sigma_{i-1}^{-1} \varrho_{i}=\varrho_{i} \lambda_{i-1, i} \varrho_{i} .
\end{aligned}
$$

Then from (25) we obtain

$$
\varrho_{i-1} \lambda_{i j} \varrho_{i-1}=\lambda_{i-1, j} .
$$

Thus, the desired relations are proven.

(iii) The first formula follows from the definitions of $\lambda_{i, i+1}$ and $\lambda_{i+1, i}$. Consider

$$
\varrho_{i} \lambda_{i j} \varrho_{i}=\varrho_{i}\left(\varrho_{j-1} \varrho_{j-2} \cdots \varrho_{i+1} \lambda_{i, i+1} \varrho_{i+1} \cdots \varrho_{j-2} \varrho_{j-1}\right) \varrho_{i} .
$$

Permuting $\varrho_{i}$ and $\lambda_{i, i+1}$ whenever possible, we obtain

$$
\varrho_{i} \lambda_{i j} \varrho_{i}=\varrho_{j-1} \cdots \varrho_{i+2}\left(\varrho_{i} \varrho_{i+1} \lambda_{i, i+1} \varrho_{i+1} \varrho_{i}\right) \varrho_{i+2} \cdots \varrho_{j-1} .
$$

Rewrite the expression in brackets as follows:

$$
\begin{aligned}
\varrho_{i} \varrho_{i+1} \lambda_{i, i+1} \varrho_{i+1} \varrho_{i} & =\varrho_{i} \varrho_{i+1} \varrho_{i}\left(\sigma_{i}^{-1} \varrho_{i+1} \varrho_{i}\right)=\varrho_{i} \varrho_{i+1}\left(\varrho_{i} \varrho_{i+1} \varrho_{i}\right) \sigma_{i+1}^{-1} \\
& =\varrho_{i} \varrho_{i+1} \varrho_{i+1} \varrho_{i} \varrho_{i+1} \sigma_{i+1}^{-1}=\varrho_{i+1} \sigma_{i+1}^{-1} .
\end{aligned}
$$

Hence,

$$
\varrho_{i} \lambda_{i j} \varrho_{i}=\varrho_{j-1} \ldots \varrho_{i+2}\left(\varrho_{i+1} \sigma_{i+1}^{-1}\right) \varrho_{i+2} \ldots \varrho_{j-1}=\lambda_{i+1, j} .
$$

Thus, the desired relations are proven.

(iv) follows from the relation $\varrho_{j-1}^{2}=e$ and the definition of $\lambda_{i j}$.

(v) is an immediate consequence of the definition of $\lambda_{i j}$.

COROLlary 1. The group $S_{n}$ acts by conjugation on the set $\left\{\lambda_{k l} \mid 1 \leq\right.$ $k \neq l \leq n\}$. This action is transitive. 
In view of Lemma 1, the subgroup $\left\langle\lambda_{k l} \mid 1 \leq k \neq l \leq n\right\rangle$ of $V P_{n}$ is normal in $V B_{n}$. Let us prove that this group coincides with $V P_{n}$ and let us find its generators and defining relations. For this purpose we use the Reidemeister-Schreier method (see, for example, [16, Ch. 2.2]).

Let $m_{k l}=\varrho_{k-1} \varrho_{k-2} \ldots \varrho_{l}$ for $l<k$ and $m_{k l}=1$ in the other cases. Then the set

$$
\Lambda_{n}=\left\{\prod_{k=2}^{n} m_{k, j_{k}} \mid 1 \leq j_{k} \leq k\right\}
$$

is a Schreier set of coset representatives of $V P_{n}$ in $V B_{n}$.

THEOREM 1. The group $V P_{n}$ admits a presentation with the generators $\lambda_{k l}, 1 \leq k \neq l \leq n$, and the defining relations

$$
\begin{aligned}
\lambda_{i j} \lambda_{k l} & =\lambda_{k l} \lambda_{i j}, \\
\lambda_{k i}\left(\lambda_{k j} \lambda_{i j}\right) & =\left(\lambda_{i j} \lambda_{k j}\right) \lambda_{k i},
\end{aligned}
$$

where distinct letters stand for distinct indices.

Proof. Define the map ${ }^{-}: V B_{n} \rightarrow \Lambda_{n}$ which takes an element $w \in V B_{n}$ to its representative $\bar{w}$ from $\Lambda_{n}$. In this case the element $w \bar{w}^{-1}$ belongs to $V P_{n}$. By Theorem 2.7 of [16] the group $V P_{n}$ is generated by

$$
s_{\lambda, a}=\lambda a \cdot(\overline{\lambda a})^{-1},
$$

where $\lambda$ runs over the set $\Lambda_{n}$ and $a$ runs over the set of generators of $V B_{n}$.

It is easy to establish that $s_{\lambda, \varrho_{i}}=e$ for all representatives $\lambda$ and generators $\varrho_{i}$. Consider the generators

$$
s_{\lambda, \sigma_{i}}=\lambda \sigma_{i} \cdot\left(\overline{\lambda \varrho_{i}}\right)^{-1} .
$$

For $\lambda=e$ we get $s_{e, \sigma_{i}}=\sigma_{i} \varrho_{i}=\lambda_{i, i+1}^{-1}$. Note that $\lambda \varrho_{i}$ is equal to $\overline{\lambda \varrho_{i}}$ in $S_{n}$. Therefore,

$$
s_{\lambda, \sigma_{i}}=\lambda\left(\sigma_{i} \varrho_{i}\right) \lambda^{-1} .
$$

From Lemma 1 it follows that each generator $s_{\lambda, \sigma_{i}}$ is equal to some $\lambda_{k l}$, $1 \leq k \neq l \leq n$. By Corollary 1 , the converse is also true, i.e., each $\lambda_{k l}$ is equal to some $s_{\lambda, \sigma_{i}}$. The first part of the theorem is proven.

To find defining relations of $V P_{n}$ we define a rewriting process $\tau$. It allows us to rewrite a word which is written in the generators of $V B_{n}$ and present an element in $V P_{n}$ as a word in the generators of $V P_{n}$. Let us associate to the reduced word

$$
u=a_{1}^{\varepsilon_{1}} a_{2}^{\varepsilon_{2}} \cdots a_{\nu}^{\varepsilon_{\nu}}, \quad \varepsilon_{l}= \pm 1, a_{l} \in\left\{\sigma_{1}, \ldots, \sigma_{n-1}, \varrho_{1}, \ldots, \varrho_{n-1}\right\},
$$

the word

$$
\tau(u)=s_{k_{1}, a_{1}}^{\varepsilon_{1}} s_{k_{2}, a_{2}}^{\varepsilon_{2}} \cdots s_{k_{\nu}, a_{\nu}}^{\varepsilon_{\nu}}
$$


in the generators of $V P_{n}$, where $k_{j}$ is a representative of the $(j-1)$ th initial segment of the word $u$ if $\varepsilon_{j}=1$, and a representative of the $j$ th initial segment of $u$ if $\varepsilon_{j}=-1$.

By [16, Theorem 2.9], the group $V P_{n}$ is defined by the relations

$$
r_{\mu, \lambda}=\tau\left(\lambda r_{\mu} \lambda^{-1}\right), \quad \lambda \in \Lambda_{n},
$$

where $r_{\mu}$ is the defining relation of $V B_{n}$.

Denote by

$$
r_{1}=\sigma_{i} \sigma_{i+1} \sigma_{i} \sigma_{i+1}^{-1} \sigma_{i}^{-1} \sigma_{i+1}^{-1}
$$

the first relation of $V B_{n}$. Then

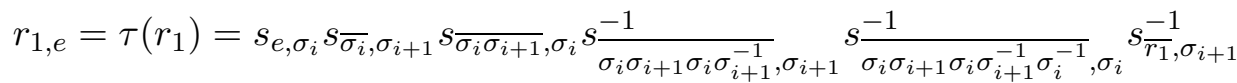

$$
\begin{aligned}
& =\lambda_{i, i+1}^{-1}\left(\varrho_{i} \lambda_{i+1, i+2}^{-1} \varrho_{i}\right)\left(\varrho_{i} \varrho_{i+1} \lambda_{i, i+1}^{-1} \varrho_{i+1} \varrho_{i}\right) \\
& \text { · }\left(\varrho_{i+1} \varrho_{i} \lambda_{i+1, i+2} \varrho_{i} \varrho_{i+1}\right)\left(\varrho_{i+1} \lambda_{i, i+1} \varrho_{i+1}\right) \lambda_{i+1, i+2} .
\end{aligned}
$$

Using the conjugating rules from Lemma 1 , we get

$$
r_{1, e}=\lambda_{i, i+1}^{-1} \lambda_{i, i+2}^{-1} \lambda_{i+1, i+2}^{-1} \lambda_{i, i+1} \lambda_{i, i+2} \lambda_{i+1, i+2} .
$$

Therefore, the relation

$$
\lambda_{i, i+1}\left(\lambda_{i, i+2} \lambda_{i+1, i+2}\right)=\left(\lambda_{i+1, i+2} \lambda_{i, i+2}\right) \lambda_{i, i+1}
$$

holds in $V P_{n}$. The remaining relations $r_{1, \lambda}, \lambda \in \Lambda_{n}$, can be obtained from this relation using conjugation by $\lambda^{-1}$. By the formulas of Lemma 1 , we obtain (27).

Let us consider the next relation of $V B_{n}$ :

$$
r_{2}=\sigma_{i} \sigma_{j} \sigma_{i}^{-1} \sigma_{j}^{-1}, \quad|i-j| \geq 2 .
$$

We have

$$
r_{2, e}=\tau\left(r_{2}\right)=s_{e, \sigma_{i}} s_{\overline{\sigma_{i}}, \sigma_{j}} s_{\overline{\sigma_{i} \sigma_{j} \sigma_{i}^{-1}}, \sigma_{i}}^{-1} s_{\overline{r_{2}}, \sigma_{j}}^{-1}=\lambda_{i, i+1}^{-1} \lambda_{j, j+1}^{-1} \lambda_{i, i+1} \lambda_{j, j+1} .
$$

Hence, the relation

$$
\lambda_{i, i+1} \lambda_{j, j+1}=\lambda_{j, j+1} \lambda_{i, i+1}, \quad|i-j| \geq 2,
$$

holds in $V P_{n}$. Conjugating this relation by all representatives from $\Lambda_{n}$, we obtain (26).

Let us prove that only trivial relations follow from all other relations of $V B_{n}$. This is evident for relations (17)-(19) defining the group $S_{n}$ because $s_{\lambda, \varrho_{i}}=e$ for all $\lambda \in \Lambda_{n}$ and $\varrho_{i}$.

Consider the mixed relation (21) (relation (20) can be considered similarly):

$$
r_{3}=\sigma_{i+1} \varrho_{i} \varrho_{i+1} \sigma_{i}^{-1} \varrho_{i+1} \varrho_{i} .
$$


Using the rewriting process, we get

$$
\begin{aligned}
r_{3, e} & =\tau\left(r_{3}\right)=s_{e, \sigma_{i+1}} s \frac{-1}{\sigma_{i+1} \varrho_{i} \varrho_{i+1} \sigma_{i}^{-1}, \sigma_{i}}=\lambda_{i+1, i+2}^{-1}\left(\varrho_{i} \varrho_{i+1} \lambda_{i, i+1} \varrho_{i+1} \varrho_{i}\right) \\
& =\lambda_{i+1, i+2}^{-1} \lambda_{i+1, i+2}=e .
\end{aligned}
$$

Thus, $V P_{n}$ is defined by relations (26)-(27).

3. The structure of the virtual braid group. From the definition of $V P_{n}$ and Lemma 1 it follows that $V B_{n}=V P_{n} \rtimes S_{n}$, i.e., $V B_{n}$ is the splittable extension of the group $V P_{n}$ by $S_{n}$. Consequently, we have to study the structure of the virtual pure braid group $V P_{n}$. Let us define the subgroups

$$
V_{i}=\left\langle\lambda_{1, i+1}, \lambda_{2, i+1}, \ldots, \lambda_{i, i+1} ; \lambda_{i+1,1}, \lambda_{i+1,2}, \ldots, \lambda_{i+1, i}\right\rangle, \quad i=1, \ldots, n-1,
$$

of $V P_{n}$. Each $V_{i}$ is a subgroup of $V P_{i+1}$. Let $V_{i}^{*}$ be the normal closure of $V_{i}$ in $V P_{i+1}$. The following theorem is the main result of this section.

THEOREM 2. The group $V P_{n}, n \geq 2$, is representable as the semi-direct product

$$
V P_{n}=V_{n-1}^{*} \rtimes V P_{n-1}=V_{n-1}^{*} \rtimes\left(V_{n-2}^{*} \rtimes\left(\ldots \rtimes\left(V_{2}^{*} \rtimes V_{1}^{*}\right)\right) \ldots\right),
$$

where $V_{1}^{*}$ is a free group of rank 2 and $V_{i}^{*}, i=2, \ldots, n-1$, are free infinitely generated subgroups.

Let us prove the theorem by induction on $n$. For $n=2$, we have

$$
V P_{2}=V_{1}=V_{1}^{*}
$$

and, by Theorem 1, the group $V_{1}$ is free generated by $\lambda_{12}$ and $\lambda_{21}$.

To make the general case clearer consider the case $n=3$.

3.1. The structure of $V P_{3}$. By Theorem 1 , the group $V P_{3}$ is generated by the subgroups $V_{1}, V_{2}$ and defined by the relations

$$
\begin{array}{ll}
\lambda_{12}\left(\lambda_{13} \lambda_{23}\right)=\left(\lambda_{23} \lambda_{13}\right) \lambda_{12}, & \lambda_{21}\left(\lambda_{23} \lambda_{13}\right)=\left(\lambda_{13} \lambda_{23}\right) \lambda_{21}, \\
\lambda_{13}\left(\lambda_{12} \lambda_{32}\right)=\left(\lambda_{32} \lambda_{12}\right) \lambda_{13}, & \lambda_{31}\left(\lambda_{32} \lambda_{12}\right)=\left(\lambda_{12} \lambda_{32}\right) \lambda_{31}, \\
\lambda_{23}\left(\lambda_{21} \lambda_{31}\right)=\left(\lambda_{31} \lambda_{21}\right) \lambda_{23}, & \lambda_{32}\left(\lambda_{31} \lambda_{21}\right)=\left(\lambda_{21} \lambda_{31}\right) \lambda_{32} .
\end{array}
$$

From these relations we obtain the next lemma.

LEMMA 2. In $V P_{3}$ the following equalities hold:

$$
\begin{array}{lll}
\lambda_{13}^{\lambda_{12}}=\lambda_{32}^{\lambda_{12}} \lambda_{13} \lambda_{32}^{-1}, & \lambda_{31}^{\lambda_{12}}=\lambda_{32} \lambda_{31} \lambda_{32}^{-\lambda_{12}}, & \lambda_{23}^{\lambda_{12}}=\lambda_{13} \lambda_{23} \lambda_{32} \lambda_{13}^{-1} \lambda_{32}^{-\lambda_{12}}, \\
\lambda_{13}^{\lambda_{12}^{-1}}=\lambda_{32}^{-1} \lambda_{13} \lambda_{32}^{\lambda_{12}^{-1}}, & \lambda_{31}^{\lambda_{12}^{-1}}=\lambda_{32}^{-\lambda_{12}^{-1}} \lambda_{31} \lambda_{32}, & \lambda_{23}^{\lambda_{12}^{-1}}=\lambda_{32}^{-\lambda_{12}^{-1}} \lambda_{13}^{-1} \lambda_{32} \lambda_{23} \lambda_{13}, \\
\lambda_{23}^{\lambda_{21}}=\lambda_{31}^{\lambda_{21}} \lambda_{23} \lambda_{31}^{-1}, & \lambda_{32}^{\lambda_{21}}=\lambda_{31} \lambda_{32} \lambda_{31}^{-\lambda_{21},}, & \lambda_{13}^{\lambda_{21}}=\lambda_{23} \lambda_{13} \lambda_{31} \lambda_{23}^{-1} \lambda_{31}^{-\lambda_{21}}, \\
\lambda_{23}^{\lambda_{21}^{-1}}=\lambda_{31}^{-1} \lambda_{23} \lambda_{31}^{\lambda_{21}^{-1}}, & \lambda_{32}^{\lambda_{21}^{-1}}=\lambda_{31}^{-\lambda_{21}^{-1}} \lambda_{32} \lambda_{31}, & \lambda_{13}^{\lambda_{21}^{-1}}=\lambda_{31}^{-\lambda_{21}^{-1}} \lambda_{23}^{-1} \lambda_{31} \lambda_{13} \lambda_{23},
\end{array}
$$

where $a^{b}$ stands for $b^{-1} a b$. 
Proof. The first and second relations of (i) immediately follow from the third and fourth relations of $V P_{3}$ (see the relations before the lemma). Similarly, the first and second relations of (ii) immediately follow from the fifth and sixth relations of $V P_{3}$.

Further, from the first and second relations of $V P_{3}$ we obtain

$$
\lambda_{23}^{\lambda_{1,2}}=\lambda_{13} \lambda_{23} \lambda_{13}^{-\lambda_{12}}, \quad \lambda_{13}^{\lambda_{21}}=\lambda_{23} \lambda_{13} \lambda_{23}^{-\lambda_{21}} .
$$

Using the already proved formulas for $\lambda_{13}^{\lambda_{12}}$ and $\lambda_{23}^{\lambda_{21}}$, we get the third formulas of (i) and (ii) respectively.

The formulas for conjugation by $\lambda_{12}^{-1}$ and $\lambda_{21}^{-1}$ can be obtained analogously.

Note that there exists an epimorphism $\varphi_{3}: V P_{3} \rightarrow V P_{2}$ which takes the generators of $V_{2}=\left\langle\lambda_{13}, \lambda_{23}, \lambda_{31}, \lambda_{32}\right\rangle$ to the unit and fixes the generators of $V_{1}=\left\langle\lambda_{12}, \lambda_{21}\right\rangle$. The kernel of this epimorphism is the normal closure of $V_{2}$ in $V P_{3}$, i.e., $\operatorname{ker}\left(\varphi_{3}\right)=V_{2}^{*}$.

Let $u$ be the empty word or a reduced word beginning with a non-zero power of $\lambda_{12}$ and representing an element from $V_{1}$. Let $\lambda_{32}(u)=\lambda_{32}^{u}=$ $u^{-1} \lambda_{32} u$. We call this element the reduced power of the generator $\lambda_{32}$ with power $u$. Analogously, if $v$ is the empty word or a reduced word beginning with a non-zero power of $\lambda_{21}$ and representing an element of $V_{1}$, then we put $\lambda_{31}(v)=\lambda_{13}^{v}$ and call it the reduced power of the generator $\lambda_{31}$ with power $v$.

LEMma 3. The group $V_{2}^{*}$ is a free group with generators $\lambda_{13}, \lambda_{23}$ and all reduced powers of $\lambda_{31}$ and $\lambda_{32}$.

Proof. To prove the lemma we can use the Reidemeister-Schreier method, but it is easier to use the definitions of normal closure and semi-direct product. Clearly, the group $V_{2}^{*}$ is generated by the elements

$$
\lambda_{13}^{w}, \lambda_{23}^{w}, \lambda_{31}^{w}, \lambda_{32}^{w}, \quad w \in V_{1} .
$$

In view of Lemma 2 , it is sufficient to take from these elements only $\lambda_{13}, \lambda_{23}$ and all reduced powers of the generators $\lambda_{31}$ and $\lambda_{32}$.

The freedom of $V_{2}^{*}$ follows from the representation of $V P_{3}$ as a semidirect product. Indeed, since $V_{1} \cap V_{2}^{*}=e, V_{1} V_{2}^{*}=V P_{3}$, it follows that $V P_{3}=V_{2}^{*} \rtimes V_{1}$. In this case the defining relations of $V P_{3}$ are equivalent to the conjugating rules of Lemma 2 . Therefore, all relations define the action of the group $V_{1}$ on the group $V_{2}^{*}$. Since there are no other relations, this means that $V_{1}$ and $V_{2}^{*}$ are free groups.

As a consequence of this lemma, we obtain the normal form of words in $V P_{3}$. Any element $w$ from $V P_{3}$ can be written in the form $w=w_{1} w_{2}$, where $w_{1}$ is a reduced word over the alphabet $\left\{\lambda_{12}^{ \pm 1}, \lambda_{21}^{ \pm 1}\right\}$ and $w_{2}$ is a reduced word over the alphabet $\left\{\lambda_{13}^{ \pm 1}, \lambda_{23}^{ \pm 1}, \lambda_{31}(u)^{ \pm 1}, \lambda_{32}(v)^{ \pm 1}\right\}$, where $\lambda_{31}(u), \lambda_{32}(v)$ are reduced powers of the generators $\lambda_{31}$ and $\lambda_{32}$ respectively. 
3.2. The proof of Theorem 2. Let $\lambda_{i j}^{*}$ denote either $\lambda_{i j}$ or $\lambda_{j i}$ from $V P_{n}$.

LEMma 4. For every $n \geq 2$ there exists a homomorphism $\varphi_{n}: V P_{n} \rightarrow$ $V P_{n-1}$ which takes the generators $\lambda_{i j}^{*}, i=1, \ldots, n-1$, to the unit and fixes the other generators.

Proof. It is sufficient to prove that all defining relations go to the defining relations under $\varphi$. For the defining relations of $V P_{n-1}$ this is evident. If the commutativity relation (see (26)) contains some generator of $V_{n-1}$ then $\varphi_{n}$ turns it into the trivial relation. Consider the left hand side of (27). We see that it contains every index two times. Hence, if this part includes some generator of $V_{n-1}$ (i.e., one of the indices is $n$ ) then some other generator involves the index $n$. Therefore, there are two generators of $V_{n-1}$ on the left hand side of the relation. Since the right hand side contains all generators from the left hand side, $\varphi_{n}$ turns this relation into the trivial relation.

Lemma 5. The following formulas are satisfied in the group $V P_{n}$ :

(i) $\lambda_{k l}^{\lambda_{i j}^{\varepsilon}}=\lambda_{k l}, \max \{i, j\}<\max \{k, l\}, \varepsilon= \pm 1$;

(ii) $\lambda_{i k}^{\lambda_{i j}}=\lambda_{k j}^{\lambda_{i j}} \lambda_{i k} \lambda_{k j}^{-1}, \lambda_{i k}^{\lambda_{i j}^{-1}}=\lambda_{k j}^{-1} \lambda_{i k} \lambda_{k j}^{\lambda_{i j}^{-1}}, i<j<k$ or $j<i<k$;

(iii) $\lambda_{k i}^{\lambda_{i j}}=\lambda_{k j} \lambda_{k i} \lambda_{k j}^{-\lambda_{i j}}, \lambda_{k i}^{\lambda_{i j}^{-1}}=\lambda_{k j}^{-\lambda_{i j}^{-1}} \lambda_{k i} \lambda_{k j}, i<j<k$ or $j<i<k$;

(iv) $\lambda_{j k}^{\lambda_{i j}}=\lambda_{i k} \lambda_{j k} \lambda_{k j} \lambda_{i k}^{-1} \lambda_{k j}^{-\lambda_{i j}}, \lambda_{j k}^{\lambda_{j i}^{-1}}=\lambda_{j k}^{-\lambda_{i k}^{-1}} \lambda_{i j}^{-1} \lambda_{j k} \lambda_{k j} \lambda_{i j}, i<j<k$ or $j<i<k$,

where, as usual, different letters stand for different indices.

Proof. (i) immediately follows from the first relation of Theorem 1.

Consider relation (27) from Theorem 1:

$$
\lambda_{k i}\left(\lambda_{k j} \lambda_{i j}\right)=\left(\lambda_{i j} \lambda_{k j}\right) \lambda_{k i} .
$$

Note that the indices of the generators are connected by one of the inequalities:
(a) $k<j<i$,
(b) $j<k<i$,
(c) $i<j<k$,
(d) $j<i<k$,
(e) $k<i<j$,
(f) $i<k<j$.

In cases (a) and (b), from (27) we obtain

which is the first formula of (ii).

$$
\lambda_{k i}^{\lambda_{k j}}=\lambda_{i j}^{\lambda_{k j}} \lambda_{k i} \lambda_{i j}^{-1},
$$

In cases (c) and (d) we obtain

$$
\lambda_{k i}^{\lambda_{i j}}=\lambda_{k j} \lambda_{k i} \lambda_{k j}^{-\lambda_{i j}}
$$

which is the first formula of (iii).

In cases (e) and (f),

$$
\lambda_{i j}^{\lambda_{k i}}=\lambda_{k j} \lambda_{i j} \lambda_{k j}^{-\lambda_{k i}}
$$


Using (ii), we obtain

$$
\lambda_{i j}^{\lambda_{k i}}=\lambda_{k j} \lambda_{i j} \lambda_{j i} \lambda_{k j}^{-1} \lambda_{j i}^{-\lambda_{k i}}
$$

which is the first formula of (iv).

The formulas for conjugations by $\lambda_{i j}^{-1}$ can be established similarly.

Assume that the theorem is proven for the group $V P_{n-1}$. Hence, any element $w \in V P_{n-1}$ can be written in the form

$$
w=w_{1} w_{2} \ldots w_{n-2}, \quad w_{i} \in V_{i}^{*},
$$

where each $w_{i}$ is a reduced word over the alphabet consisting of the generators $\lambda_{k i}^{ \pm 1}, 1 \leq k \leq i-1$, and reduced powers of the generators $\lambda_{k i}$, $1 \leq k \leq i-1$, and their inverses. Let us define reduced powers of generators in the group $V_{n-1}^{*}$. We say that the element $\lambda_{n k}(w)=\lambda_{n k}^{w}$ is the reduced power of the generator $\lambda_{n k}$ if $w$ is the empty word or a word written in the normal form and beginning with a reduced power of some generator $\lambda_{l k}$ or its inverse.

The statement about decomposition as the semi-direct product $V P_{n}=$ $V_{n}^{*} \rtimes V P_{n-1}$ is quite evident. It remains to find generators of $V_{n}^{*}$ and prove its freedom.

Lemma 6. The group $V_{n-1}^{*}$ is free. It is generated by $\lambda_{1 n}, \lambda_{2 n}, \ldots, \lambda_{n-1, n}$ and all reduced powers of the generators $\lambda_{n 1}, \lambda_{n 2}, \ldots, \lambda_{n, n-1}$.

Proof. The proof is similar to that of Lemma 3. From Lemma 5 it follows that the indicated elements generate $V_{n-1}^{*}$. Further, since the set of defining relations of $V P_{n}$ is equivalent to the set of conjugating formulas defining the action of $V P_{n-1}$ on $V_{n-1}^{*}$, only trivial relations are satisfied in $V_{n-1}^{*}$.

Theorem 2 follows from these results.

As a consequence, we obtain the normal form of words in $V B_{n}$.

CoRollary 2. Every element of $V B_{n}$ can be uniquely written in the form

$$
w=w_{1} \ldots w_{n-1} \lambda, \quad \lambda \in \Lambda_{n}, w_{i} \in V_{i}^{*},
$$

where $w_{i}$ is a reduced word in the generators, reduced powers of the generators and their inverses.

The homomorphism defined above of the virtual braid group onto the welded braid group agrees with the decomposition from Theorem 2 and with the decomposition of $C_{n} \simeq W B_{n}$ described in the first section.

COROLlary 3. The homomorphism $\varphi_{V W}: V B_{n} \rightarrow W B_{n}$ agrees with the decompositions of these groups, i.e., it maps $V P_{n}$ onto $C b_{n} \simeq W P_{n}$ and the factors $V_{i}^{*}$ onto the factors $D_{i}, i=1, \ldots, n-1$. 
4. The universal braid group. Let us define the universal braid group $U B_{n}$ as the group with generators $\sigma_{1}, \ldots, \sigma_{n-1}, c_{1}, \ldots, c_{n-1}$, defining relations (1)-(2), the relations

$$
c_{i} c_{j}=c_{j} c_{i}, \quad|i-j| \geq 2,
$$

and the mixed relations

$$
c_{i} \sigma_{j}=\sigma_{j} c_{i}, \quad|i-j| \geq 2 .
$$

Recall (see [6]) that Artin's group of the type $I$ is the group $A_{I}$ with generators $a_{i}, i \in I$, and defining relations

$$
a_{i} a_{j} a_{i} \ldots=a_{j} a_{i} a_{j} \ldots, \quad i, j \in I,
$$

where the words on the left and right hand sides consist of $m_{i j}$ alternating letters $a_{i}$ and $a_{j}$.

Proposition 1. (i) $U B_{n}$ has the braid group $B_{n}$ as a subgroup.

(ii) There exist surjective homomorphisms

$$
\varphi_{U S}: U B_{n} \rightarrow S G_{n}, \quad \varphi_{U V}: U B_{n} \rightarrow V B_{n}, \quad \varphi_{U B}: U B_{n} \rightarrow B_{n} .
$$

(iii) $U B_{n}$ is Artin's group.

Proof. (i) Evidently, there exists a homomorphism $B_{n} \rightarrow U B_{n}$. On the other hand, setting $\psi\left(\sigma_{i}\right)=\sigma_{i}, \psi\left(c_{i}\right)=e, i=1, \ldots, n-1$, we obtain a retraction $\psi$ of $U B_{n}$ onto $B_{n}$. Therefore, the subgroup $\left\langle\sigma_{1}, \ldots, \sigma_{n-1}\right\rangle$ of $U B_{n}$ is isomorphic to the braid group $B_{n}$.

(ii) Define the map $\varphi_{U S}$ as follows:

$$
\varphi_{U S}\left(\sigma_{i}\right)=\sigma_{i}, \quad \varphi_{U S}\left(c_{i}\right)=\tau_{i}, \quad i=1, \ldots, n-1 .
$$

Comparing the defining relations of $U B_{n}$ and $S G_{n}$, we see that this map is a homomorphism. Analogously, we can show that the map

$$
\sigma_{i} \mapsto \sigma_{i}, \quad c_{i} \mapsto \varrho_{i},
$$

extends to a homomorphism $\varphi_{U V}$, and the map

$$
\sigma_{i} \mapsto \sigma_{i}, \quad c_{i} \mapsto e,
$$

extends to a homomorphism $\varphi_{U B}$.

(iii) immediately follows from the defining relations of $U B_{n}$ and the definition of Artin's group.

It should be noted that none of the groups $S G_{n}, V B_{n}, W B_{n}$ (in the natural presentations) is Artin's group.

The following questions naturally arise in the context of the results obtained above. 
Problems. (i) Solve the word and conjugacy problems in $U B_{n}, n>2$.

(ii) Is it possible to give some geometric interpretation for elements of $U B_{n}$ similar to the geometric interpretation for elements of the braid groups $B_{n}, S G_{n}, V B_{n}, U B_{n}$ ?

\section{References}

[1] J. C. Baez, Link invariants of finite type and perturbation theory, Lett. Math. Phys. 26 (1992), 43-51.

[2] V. G. Bardakov, The structure of a group of conjugating automorphisms, Algebra Logika 42 (2003), 515-541 (in Russian).

[3] - , The structure of the group of conjugating automorphisms and the linear representation of the braid groups of some manifolds, preprint (math.GR/0301247).

[4] J. S. Birman, Braids, Links and Mapping Class Groups, Princeton Univ. Press and Univ. of Tokyo Press, 1974.

[5] -, New points of view in knot theory, Bull. Amer. Math. Soc. 28 (1993), 253-287.

[6] E. Brieskorn und K. Saito, Artin-Gruppen und Coxeter-Gruppen, Invent. Math. 17 (1972), 245-271.

[7] R. Corran, A normal form for a class of monoids including the singular braid monoids, J. Algebra 223 (2000), 256-282.

[8] O. T. Dasbach and B. Gemein, The word problem for the singular braid monoid, preprint, 1999.

[9] R. Fenn, E. Keyman and C. Rourke, The singular braid monoid embeds in a group, J. Knot Theory Ramifications 7 (1998), 881-892.

[10] R. Fenn, R. Rimányi and C. Rourke, The braid-permutation group, Topology 36 (1997), 123-135.

[11] B. Gemein, Singular braids and Markov's theorem, J. Knot Theory Ramifications 6 (1997), 441-454.

[12] M. Goussarov, M. Polyak and O. Viro, Finite-type invariants of classical and virtual knots, Topology 39 (2000), 1045-1068.

[13] M. Gutiérrez and S. Krstić, Normal forms for basis-conjugating automorphisms of a free group, Int. J. Algebra Comput. 8 (1998), 631-669.

[14] S. Kamada, Braid presentation of virtual knots and welded knots, preprint (math.GT /0008092).

[15] L. H. Kauffman, Virtual knot theory, European J. Combin. 20 (1999), 663-690.

[16] W. Magnus, A. Karrass and D. Solitar, Combinatorial Group Theory, Interscience Publ., New York, 1996.

[17] A. A. Markov, Foundations of the algebraic theory of braids, Trudy Mat. Inst. Steklova 16 (1945), 1-54 (in Russian).

[18] J. McCool, On basis-conjugating automorphisms of free groups, Canad. J. Math. 38 (1986), 1525-1529.

[19] A. G. Savushkina, On the group of conjugating automorphisms of a free group, Math. Notes 60 (1996), 68-80; translation from Mat. Zametki 60 (1996), 92-108.

[20] V. A. Vassiliev, Complements of Discriminants of Smooth Maps: Topology and Applications, Transl. Math. Monogr. 98, Amer. Math. Soc., Providence, RI, 1992.

[21] V. V. Vershinin, On homology of virtual braids and Burau representation, J. Knot Theory Ramifications 10 (2001), 795-812. 
[22] V. V. Vershinin, On the singular braid monoid, preprint (math.GR/0309339).

Sobolev Institute of Mathematics

Novosibirsk 630090, Russia

E-mail: bardakov@math.nsc.ru

Received 15 March 2004;

in revised form 26 August 2004 